\title{
Hydrothermal waves and corotating rolls in laterally heated convection in simple liquids
}

\author{
Carlos Pérez-García ${ }^{1 *}$, Santiago Madruga1, Blas Echebarria ${ }^{2}$, Georgy Lebon ${ }^{3}$ and \\ Javier Burguete ${ }^{1}$ \\ 1 Instituto de Física, Universidad de Navarra, Pamplona, Spain \\ ${ }^{2}$ Departament de Física Aplicada, Universitat Politècnica de Catalunya, Barcelona, Spain \\ ${ }^{3}$ Université de Liège, Institut de Physique B5, Liège, Belgium \\ *Corresponding author (carlos@fisica.unav.es) \\ PACS numbers: 47.20.Dr, 47.20.Bp, 47.54.+r, 47.27.Te, 44.25.+f, 47.20.Ma
}

\begin{abstract}
The stability of a liquid layer with an undeformable interface open to the atmosphere, subjected to a horizontal temperature gradient, is theoretically analysed. Buoyancy and surface tension forces give rise to a basic flow for any temperature difference applied on the system. Depending on the liquid depth, this basic flow is destabilised either by an oscillatory instability, giving rise to the so-called hydrothermal waves, or by a stationary instability leading to corotating rolls. Oscillatory perturbations are driven by the basic flow and therefore one must distinguish between convective and absolute thresholds. The instability mechanisms as well as the different regimes observed in experiments are discussed. The calculations are performed for a fluid used in recent experiments, namely silicone oil of $0.65 c S t(\operatorname{Pr}=10)$. In particular, it is shown that two branches of absolute instability exist, which may be related to the two types of hydrothermal waves observed experimentally.
\end{abstract}

\section{Introduction}

Convection constitutes one of the most distinguished physical phenomena to probe new ideas about dynamical systems. It has been particularly useful to study pattern formation, wavenumber selection mechanisms, the dynamics of defects, etc. [1]. Convection in simple liquids leads usually to steady motions, which organise themselves into cellular patterns, as observed by Bénard [2] more than a century ago. The primary formation mechanism of these patterns lies in the fact that temperature perturbations along the free surface cause surface tension variations which set the liquid interface into motion. Because of the liquid viscosity, the moving interface gives rise to shear stresses which drive a bulk flow. This flow is usually called thermocapillary 
convection or Marangoni convection and can arise in liquid-gas or liquid-liquid interfaces under a temperature gradient. For temperature gradients normal to the layer, stationary Bénard cells are displayed, but under lateral heating, thermocapillarity may also induce wave motions. This phenomenon can be relevant in many processes of technological interest, for example in floating zone crystal growth, thin-film coating, thermocapillary migration of small droplets or bubbles, electron beam vaporisation and laser welding.

In laterally heated systems, the instability mechanism is quite complex. A basic flow settles down in the fluid as soon as lateral heating is applied, and the resulting temperature profile is non-linear. This flow may be destabilised by the so-called hydrothermal waves after a critical temperature gradient is reached. Smith and Davis [3] discussed this stability problem by considering thermocapillary flows only. Furthermore, Smith $[4,5]$ showed the existence of two different mechanisms of instability depending on the Prandtl number $P r$. At low $P r$, the energy necessary to sustain disturbances comes from the horizontal applied temperature field and hydrothermal waves propagate in a direction perpendicular to the horizontal temperature gradient. At high $\mathrm{Pr}$, the energy is extracted from the vertical temperature field by vertical convection and it gives rise to hydrothermal waves propagating parallel to the temperature gradient. At intermediate $P r$, the mechanism is a combination of the previous effects and leads to waves forming an angle with the streamwise direction.

But thermocapillary convection is usually mixed with buoyancy effects. Laure and Roux [6], Ben Hadid and Roux [7] considered thermocapillary and buoyancy effects in liquids with a small Prandtl number $(\operatorname{Pr}<1)$. Later on, Gershuni et al. [8] found steady rolls in the case of conducting surfaces for $\operatorname{Pr}>1$. Parmentier et al. [9] and Mercier and Normand [10] performed calculations by taking into account buoyancy effects and thermal transfer properties at the interface, and confirmed theoretically the existence of oblique hydrothermal waves for intermediate $P r$.

On the experimental side, Schwabe et al. [11] observed oscillatory motions in half zones. Villers and Platten [12] reported oscillatory motions in experiments and numerical simulations in a shallow layer of acetone $(P r=4.2)$ in a rectangular container submitted to a horizontal temperature gradient along the larger extension. As the heating increased they also found a transition to a multicellular flow, composed by corotating rolls, the intensity of which decreases as one moves away from the hot wall. Similar results were obtained by Ezersky et al. [13] and de Saedeleer et al. [14]. The first observations of hydrothermal waves were reported by Daviaud and Vince [15] who used a shallow layer of silicone oil of $0.65 c S t(P r=10)$ in a narrow rectangular channel heated horizontally across the shorter side. These observations were completed by Muckolobwiez et al. [16] in an annular geometry. Moreover, Riley and Neitzel [17] confirmed regimes of hydrothermal waves, steady multicellular flow, and oscillatory multicellular flows in silicone oil of $1 c S t(P r=13.9)$ in a wide rectangular container. These results were interpreted some years later by Priede and Gerbeth [18] in terms of absolute and convective instability.

More recently Pelacho et al. [19] and Burguete et al. [20] reported new measurements on hydrothermal waves in rectangular containers (see Figure 1). As quoted in [20], 


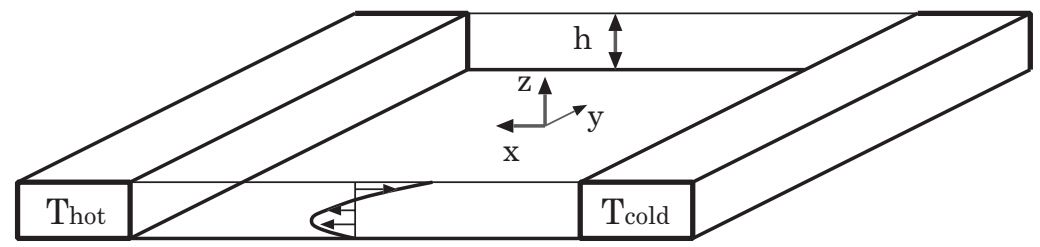

Figure 1 Scheme of a lateral heating set-up used in experiments.

important discrepancies regarding either the convective threshold or the critical wavenumber still remain. Moreover, Schwabe et al. [11], Burguete et al. [20], Garnier [21], Garnier et al. [22, 23] find two wave regimes, one for very thin layers (typically $h<1.4 \mathrm{~mm}$ ) and another for thicker layers. Finally, Shetsova et al. [24] investigated numerically a finite two-dimensional cavity heated from the side, and analysed the interplay between the steady cell created near the hot wall and the hydrothermal waves.

The aim of this article is to complete the linear stability analysis of a basic flow for a liquid with an intermediate Prandtl number, $P r=10$, by varying the liquid depth, as generally considered in experiments, and to discuss the nature of the instability leading to hydrothermal waves or stationary rolls. The paper is organised as follows. In Section 2 are established the governing equations and boundary conditions. A linear stability analysis is performed in Section 3. The main results of the stability analysis and comparison with experiments are reported in Section 4; final conclusions are drawn in Section 5.

\section{Governing equations and boundary conditions}

We consider a horizontal liquid layer of thickness $h$ and infinite horizontal extent under a horizontal temperature gradient. As usual, we introduce thermocapillary effects through the temperature-dependent surface tension

$$
\sigma(T)=\sigma\left(T_{o}\right)+\frac{\partial \sigma}{\partial T}\left(T-T_{o}\right)
$$

The system is heated with a constant temperature gradient $\beta$ imposed along the horizontal direction, which produces a conducting temperature profile given by

$$
T=T_{-}+\beta x
$$

where $T_{-}$is the temperature of the cold side. The properties of the liquid are: density $\rho$, kinematic viscosity $v$, coefficient of volume expansion $\alpha$, thermal conductivity $\lambda$, and thermal diffusivity $\kappa$; acceleration of gravity, pressure and velocity are denoted as $g, p$, and $\mathbf{u}=(u, v, w)$, respectively. As scales of length, time, velocity, pressure, and temperature we take $h, h^{2} / \kappa, \kappa / h, \rho v \kappa / h^{2}$, and $\beta h$, respectively. The dimensionless mass, momentum and energy balance equations are 


$$
\begin{aligned}
& \nabla \cdot \mathbf{u}=0 \\
& \operatorname{Pr}^{-1}\left[\partial_{t} \mathbf{u}+(\mathbf{u} \cdot \nabla) \mathbf{u}\right]=-\nabla p+\operatorname{RaT} \hat{\mathbf{z}}+\nabla^{2} \mathbf{u}, \\
& \partial_{t} T+\mathbf{u} \cdot \nabla T=\nabla^{2} T
\end{aligned}
$$

in which the Oberbeck-Boussinesq approximation has been assumed and $\hat{\mathbf{z}}$ stands for the unit vector in the $z$-direction. The dimensionless boundary conditions are

- rigid conducting bottom wall $(z=0)$

$$
\mathbf{u}=0 \quad T=T_{-}+x
$$

- free surface $(z=1)$

$$
\begin{aligned}
& w=0, \\
& \partial_{z} \mathbf{u}_{h}=-M a \nabla_{h} T, \\
& \partial_{z} T=-B i\left(T-T_{\infty}(x)\right),
\end{aligned}
$$

where $T_{\infty}(x)$ denotes the reference temperature in the air far from the interface, $\mathbf{u}_{h}$ the horizontal velocity, $\nabla_{h}$ the horizontal gradient. The following nondimensional numbers have also been introduced:

- the Rayleigh number $R a=\frac{\alpha g h^{4} \beta}{v \kappa}$ (buoyancy effects),

- the Marangoni number $M a=\frac{-(d \sigma / d T) h^{2} \beta}{\rho v \kappa}$ (thermocapillary effects),

- the Prandtl number $\operatorname{Pr}=\frac{v}{\kappa}$ (ratio of viscous over thermal diffusive time scales), and

- the Biot number $B i=\frac{h d}{\lambda}$, where $h$ stands for the thermal exchange coefficient across the free interface and $\lambda$ is the thermal conductivity of the liquid.

The ratio between the Rayleigh $R a$ and Marangoni $M a$ numbers is the dynamic Bond number

$$
B o=\frac{R a}{M a}=\frac{g \alpha \rho h^{2}}{|\partial \sigma / \partial T|},
$$

which depends on the liquid depth for a given liquid.

\section{Linear stability analysis}

\subsection{The basic state}

When a horizontal temperature gradient is applied, the liquid develops a basic flow parallel to the flat interface [3]. Usually, the basic flow is a monocellular flow that 
goes upwards at the hot sidewall and downwards at the cold sidewall (see Figure 1). In the present theoretical analysis, the liquid layer is assumed to be of infinite horizontal extent, but the horizontal temperature gradient remains finite. Under these assumptions the reference state is characterised by a velocity field of the form $\mathbf{u}=\left(u_{0}(z), 0,0\right)$ and the temperature distribution given by $T-T_{-}=x+\tau_{0}(z)$.

After introducing these expressions in Eqs. (3)-(5) and applying the return condition, that is, imposing that the mass flow across any vertical section must vanish,

$$
\int_{0}^{1} u_{0} d z=0
$$

one arrives after integration at the following expressions for the horizontal velocity and temperature profiles [10]:

$$
\begin{aligned}
& u_{0}=\operatorname{BoMa}\left(8 z^{3}-15 z^{2}+6 z\right) / 48-\operatorname{Ma}\left(3 z^{2}-2 z\right) / 4 \\
& \tau_{0}=\operatorname{BoMaz}\left(8 z^{4}-25 z^{3}+20 z^{2}-3 B i^{\dagger}\right) / 960-\operatorname{Maz}\left(3 z^{3}-4 z^{2}+B i^{\dagger}\right) / 48
\end{aligned}
$$

with $B i^{\dagger}=B i /(1+B i)$. These profiles are composed of two terms, one due to buoyancy and another to thermocapillary effects. A scheme of these profiles is drawn in Figure 2.

Let us remark that this idealised basic flow should differ from the return flow in any experiment in which sidewalls will change these profiles [24]. Nevertheless, experimental flow far from the sidewalls should not differ substantially from that idealised flow in sufficiently wide systems.

\subsection{Perturbation equations}

The next step consists in performing a linear stability analysis of the basic flow against infinitesimal perturbations of velocity $\mathbf{u}=\left(u_{0}(z)+u^{\prime}, v^{\prime}, w^{\prime}\right)$, temperature $T=x+\tau_{0}(z)+\theta^{\prime}$ and pressure $p=p_{0}+p^{\prime}$ that must obey
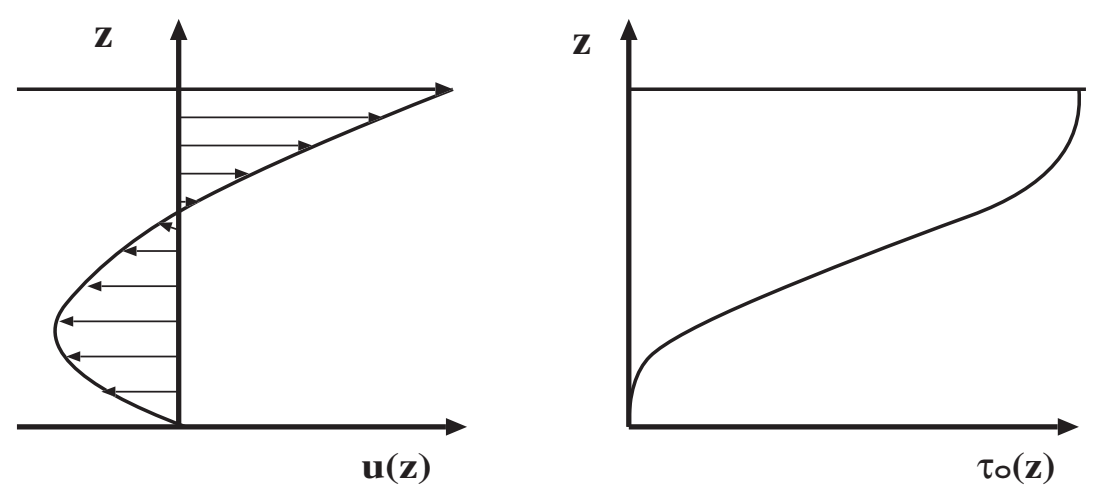

Figure 2 Scheme of the velocity and temperature profiles of the basic flow. 


$$
\begin{aligned}
& \nabla \cdot \mathbf{u}=0 \\
& \operatorname{Pr}^{-1}\left[\partial_{t} \mathbf{u}+u_{0} \partial_{x} \mathbf{u}+w \partial_{z} u_{0} \hat{\mathbf{x}}\right]=-\nabla p+B o M a \theta \hat{\mathbf{z}}+\nabla^{2} \mathbf{u} \\
& \frac{\partial \theta}{\partial t}+u_{0} \partial_{x} \theta+w \partial_{z} \tau+u=\nabla^{2} \theta
\end{aligned}
$$

in which the primes have been deleted for clarity and $\hat{\mathbf{x}}$ denotes the unit vector in the $x$-direction. The boundary conditions for these perturbations are

- at $z=0$ (rigid conducting bottom)

$$
\mathbf{u}=0 \quad \theta=0
$$

- at $z=1$

$$
\partial_{z} \mathbf{u}_{h}-M a \nabla_{h} \theta=\partial_{z} \theta+B i \theta=w=0
$$

As usual, the disturbed fields are expanded in normal modes, that is,

$$
(\mathbf{u}, \theta, p)=[\mathbf{U}(z), \Theta(z), P(z)] \exp i(\mathbf{k} \cdot \mathbf{x}-\omega \mathbf{t}),
$$

where $\omega$ denotes the complex time growth rate, $k_{x}$ the wavenumber in the streamwise direction and $k_{y}$ the wavenumber in the spanwise direction. Eliminating the pressure within the balance equations, the equations for the amplitude of the perturbations $U, W, \Theta$ read as

$$
\begin{aligned}
& D^{2} U=L U+\frac{k_{y}^{2}}{k^{2}} \operatorname{Pr}^{-1} W D u_{0}+i \frac{k_{x}}{k^{2}}\left(D^{3} W-L D W\right), \\
& D^{4} W=\left(L+k^{2}\right) D^{2} W-L k^{2} W+\operatorname{BoMak}^{2} \Theta-i k_{x} \operatorname{Pr}^{-1} W D^{2} u_{0}, \\
& D^{2} \Theta=\left(k^{2}+i\left(\omega+k_{x} u_{0}\right)\right) \Theta+U+W D \tau_{0},
\end{aligned}
$$

where $D=\frac{d}{d z}$, and $\left.L=k^{2}+i P_{r}^{-1}\left(\omega+k_{x} u_{0}\right)\right)$. The corresponding relevant boundary conditions are

- at $z=0, U=W=D W=0$, and $\Theta=0$,

- at $z=1, W=D \Theta+B i \Theta=D U+i k_{x} M a \Theta=D^{2} W+M a k^{2} \Theta=0$.

\subsection{Method of solution}

The linear analysis leads to an eigenvalue problem in the form

$$
\boldsymbol{A} X=\omega \boldsymbol{B} X
$$


wherein the complex growth rate $\omega$ is the eigenvalue and $X$ the eigenvector. The set of equations (14) is solved with a pseudospectral method, the tau-Chebyshev method [25], in which the unknowns $X$ are expanded in terms of Chebyshev polynomials $T_{n}(z)$ :

$$
X(z) \approx \sum_{k=0}^{N} a_{k} T_{k}(z)
$$

Finally, one obtains a dispersion relation of the form $\omega(k, M a, B o)$. Before discussing the results, let us briefly introduce the notions of convective and absolute instabilities.

\subsection{Convective/absolute instability}

This linear stability analysis is more involved than in the case of a fluid layer subject to a vertical temperature gradient, because any perturbation which becomes unstable is dragged by the basic flow while still growing. If at a fixed location in the laboratory frame, the perturbation decays to zero in the long term, while growing exponentially in some moving frame, the flow is said to be convectively unstable. In the presence of convective instability, infinitesimal disturbances may leave a system of finite length before reaching an observable value. When a perturbation grows at any point in space, it is called absolutely unstable. Absolute instability can be treated by considering perturbations in the form of wavepackets driven by the flow, that is, by assuming complex wavenumbers. Consequently, besides the usual time growth, the spatial growth must also be considered. A general discussion on this topic can be found in the review papers by Briggs [26], Bers [27], and Huerre and Monkewitz [28].

Finding the absolute instability threshold relies basically on a criterion defining the direction of propagation. This direction may be correctly determined by the sign of the real part of the group velocity [18]. Consequently, the wavenumber $k_{0}$ corresponding to a vanishing group velocity

$$
\frac{\partial \omega}{\partial k}\left(k_{0}\right)=0
$$

has to be tracked. If a complex frequency $\omega_{0}=\omega\left(k_{0}\right)$ has a positive growth rate, that is, the imaginary part $\omega_{0, i} \geq 0$, the flow becomes absolutely unstable. The value $k_{0}$ corresponding to a saddle point of the dispersion relation $\omega(k)$, is defined by the two conditions

$$
\frac{\partial \omega_{r}}{\partial k_{r}}=\frac{\partial \omega_{i}}{\partial k_{r}}=0
$$

These two conditions are often difficult to evaluate, but they can be rewritten in terms of the marginal curves by means of the following trick. The frequency $\omega$ 
depends on the wavenumber $k$, and the Marangoni number can be considered as a parameter, so that $\omega=\omega(k ; M a)$. An additional restriction results from the neutral stability condition $\omega_{i}(k ; M a)=0$, which defines implicitly the critical Marangoni number $M a=M a\left(k_{r} ; k_{i}\right)$. Partial differentiation with respect to $k_{r}$ gives $\frac{\partial \omega_{i}}{\partial k_{r}}+$ $\frac{\partial \omega_{i}}{\partial M a} \frac{\partial M a}{\partial k_{r}}=0$. The neutral stability curve $\frac{\partial \omega_{i}}{\partial M a}>0$ and the condition for the saddle point $\frac{\partial \omega_{i}}{\partial k_{r}}=0$ imply that $\frac{\partial M a}{\partial k_{r}}=0$, and that $M a$ should be a local extremum. In a representation of $M a$ as a function of $\omega_{r}$ the conditions (17) are equivalent to a cusp (pinching) point. We use this property to determine the absolute instability threshold.

\subsection{Stationary longitudinal rolls}

At this point, some remarks are in form concerning stationary solutions. The standard linear theory predicts stable stationary solutions only when unrealistic (very high $\mathrm{Bi}$ ) heat transfer coefficients are considered [10]. In these theoretical works, the layer is assumed to be of infinite horizontal extension and therefore only intrinsic perturbations are considered. However, numerical simulations [6] and experiments [11-14] show that a pattern of corotating stationary rolls transverse to the basic flow may appear in the liquid. This apparent contradiction is resolved taking into account that the boundary layer on the hot sidewall constitutes a finite perturbation that, depending on the sign of the attenuation rate of the perturbation, may spatially fade, that is, remain localised, or become amplified spreading throughout the layer. The basic flow is in the $x$-direction, and therefore only the imaginary part of $k_{x}$ has to be considered in the linear equations and boundary conditions. Therefore, the limit of these two situations is given by the conditions $\omega_{r}(k, M a)=0$, and $\omega_{i}(k, M a)=0$ with $k_{x i}=0$.

\section{Results and comparison with experiments}

In Figures 3 and 4 are represented the main results of the linear stability analysis. We have also compared our results with the experimental data obtained by Pelacho et al. [19] and Burguete et al. [20] for $0.65 c S t$ silicone oil $(\operatorname{Pr}=10)$. All our calculations are performed for this fluid, by assuming $B i=1$. In Figure 3 a the critical Marangoni number as a function of $B o$ is presented. Thresholds for the absolute, convective and stationary instability are given by the solid thick, dashed-dotted and dotted lines, respectively. The curves for the convective and stationary instabilities are similar to those reported by Priede and Gerbeth [18] for $P r=13.9$. The curve for the absolute instability presents two branches that could be related with the two wave regimes reported by Burguete et al. [20] in narrow rectangular enclosures, and by Garnier and Chiffaudel [22] in annular containers. From Figure 3, it becomes apparent that for $B o \leq 0.3$ convective and absolute thresholds are close to each other and corotating rolls should not appear. For $B o \geq 0.3$ the threshold values for the convective and absolute instability diverge as $B o$ is increased. Moreover the stationary solution pre- 


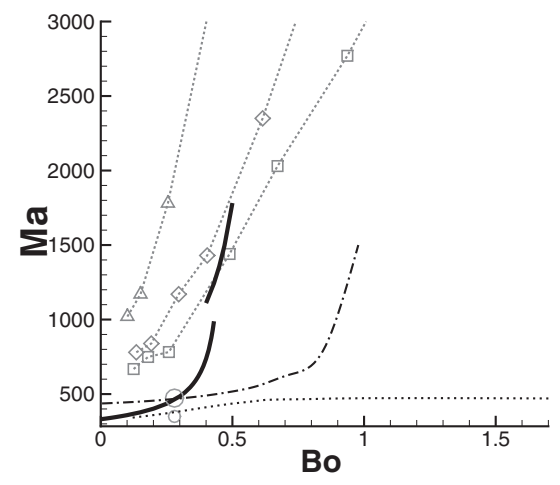

(a)

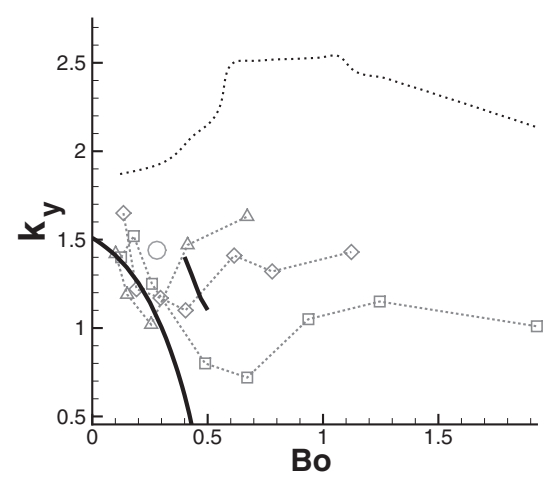

(b)

Figure 3 Critical Marangoni numbers (a) and transversal wave-numbers (b) as functions of the dynamic Bond number $B o$. Experimental data for: $L_{x}=10 \mathrm{~mm}, \triangle, L_{x}=20 \mathrm{~mm}, \diamond$, $L_{x}=30 \mathrm{~mm}, \square[20]$. The circles $\circ$ indicate the experimental values in [19]. Solid thick lines: absolute branches. Dot-dashed line: stationary threshold. Dotted line: convective instability.

cedes the absolute instability in this regime. Measurements in [20] were performed in containers with $1 \mathrm{~cm}, 2 \mathrm{~cm}$, and $3 \mathrm{~cm}$ width.

As expected, experimental results in the widest cell $(3 \mathrm{~cm})$ are the closest to the theoretical thresholds. Results by Pelacho et al. [19] in a wide container $(8 \mathrm{~cm})$ are represented by circles in Figures 3 and 4. In Figure 3a the upper circle corresponds to the critical Marangoni number calculated with the temperature difference applied upon the layer, while the lower circle stands for $M a$ obtained from the temperature difference measured on the surface in the central part of the layer. We notice that our
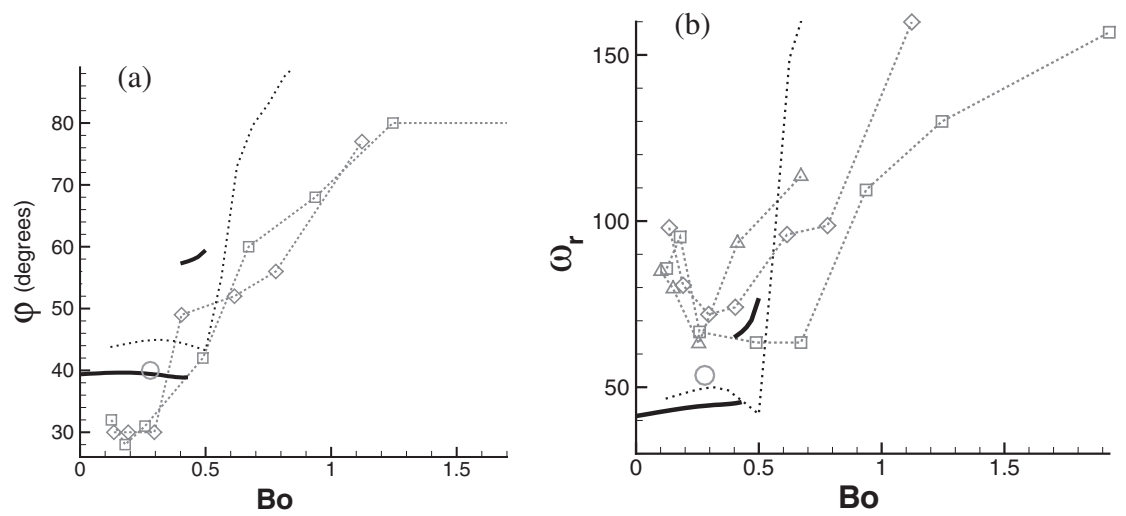

Figure 4 Angle of propagation (a) and frequency (b) of the transverse waves as functions of the dynamic Bond number Bo. Experimental data for: $L_{x}=10 \mathrm{~mm}, \triangle, L_{x}=20 \mathrm{~mm}, \diamond$, $L_{x}=30 \mathrm{~mm}, \square[20]$. The circles $\circ$ indicate the experimental values in [19]. Solid thick lines: absolute branches. Solid thin line: convective instability. 
theoretical results agree qualitatively with the experimental values, which are nevertheless always greater than the theoretical ones, owing to the thermal boundary layer on the sidewalls not taken into account in an idealised model.

But the most interesting result concerns the spanwise wavenumber $k_{y}$ in Figure $3 \mathrm{~b}$. As reported by Burguete et al. [20], a discrepancy of a factor of two between the measured values of $k_{y}$ and the theoretical values predicted from the convective instability is apparent. However, the absolute instability curve is closer to the experimental values than the convective one. Here again two absolute branches are observed. In each branch, $k_{y}$ decreases when $B o$ is increased, but it jumps from a branch to the other. This suggests again that these branches correspond to two different wave regimes. To the best of our knowledge, this is the first time that the occurrence of such regimes is discussed.

In Figure 4 are gathered the calculated values for the angle of propagation (with respect to the direction of the basic flow) and the frequency of the waves. It is seen that absolute branches behave differently with $B o$ : the angle is almost constant when the surface tension is dominant, and decreases when buoyancy becomes important. A reasonable agreement between theory and experiments is also achieved for these two quantities.

\section{Conclusions and discussion}

Thermo-convective instability of a liquid layer under a horizontal temperature gradient has been theoretically studied. It is confirmed that travelling hydrothermal waves and stationary corotating rolls are possible solutions of this problem. Our analysis has shown that the two regimes of travelling waves reported in experiments by Burguete et al. [20] and Garnier and Chiffaudel [22] may be associated with two branches of the absolute instability. It is also argued that the discrepancy between theory and experiments regarding the spanwise wavevector reported by Burguete et al. [20] may be explained by admitting that these data correspond to an absolute instability, instead of a convective instability, as assumed in previous analyses [10]. The nature of the results obtained in the present work clearly indicate that the problem of lateral heating convection constitutes an interesting prototype to study propagation and interaction of waves in simple liquids.

\section{Acknowledgements}

We thank A. Chiffaudel, F. Daviaud, N. Garnier, M.A. Pelacho, and J. Priede for interesting discussions. This work has been partially supported by the DGICYT (Spanish Government) under grant BFM2002-O2011, the PIUNA (Universidad de Navarra), and by a Spain-France Integrated Action (HF2001-008). B.E. wish to thank the MEC (Spanish Government) for a contract within the "Ramón y Cajal" Program. One of us (G.L.) acknowledges the support of ESA Prodex VII and CIMEX II projects. 


\section{References}

[1] Cross, M.C., Hohenberg, P.C., Pattern formation outside of equilibrium, Rev. Mod. Phys., 65 (1993), 852-1112.

[2] Bénard, H., Les tourbillons cellulaires dans une nappe liquide transportent la chaleur par convection en régime permanent, Rev. Gén. Sci. Pure Appl., 11 (1900), 1261-1271, 13091318.

[3] Smith, M.K., Davis, S.H., Instabilities of dynamic thermocapillary liquid layers. Part 1. Convective instabilities, J. Fluid Mech., 132 (1983), 119-144.

[4] Smith, M.K., Instability mechanisms in dynamic thermocapillary liquid layer, Phys. Fluids, 29 (1986), 3182-3186.

[5] Smith, M.K., The nonlinear stability of dynamic thermocapillary liquid layers, J. Fluid Mech., 194 (1988), 391-415.

[6] Laure, P., Roux, B., Linear and nonlinear analysis of the Hadley circulation, J. Crys. Growth, 97 (1989), 226-234.

[7] Ben Hadid, H., Roux, B., Thermocapillary convection in long layers of low-Prandtlnumber melts subjected to a horizontal temperature gradient, J. Fluid Mech., 221 (1990), 77-103; Buoyancy and thermocapillary-driven flows in differentially heated cavities for low-Prandtl-number fluids, J. Fluid Mech., 235 (1992), 1-36.

[8] Gershuni, G.Z., Laure, P., Myznikov, V.M., Roux, B., Zhukhovitsky, E.M., On the stability of plane-parallel advective flows in long horizontal layers, Micrograv. Q., 2 (1992), $141-152$.

[9] Parmentier, P.M., Regnier, V.C., Lebon, G., Buoyant-thermocapillary instabilities in medium $\operatorname{Pr}$ number fluid layers subject to a horizontal temperature gradient, Int. J. Heat Mass Transfer, 36 (1993), 2417-2427.

[10] Mercier, J.F., Normand, C., Buoyant-thermocapillary instabilities of differentially heated liquid layers, Phys. Fluids, 8 (1996), 1434-1445.

[11] Schwabe, D., Müller, U., Schneider, J., Scharmann, A., Instabilities of shallow dynamic thermocapillary liquid bridges, Phys. Fluids A, 4 (1992), 2368-2381.

[12] Villers, D., Platten, J.K., Coupled buoyancy and Marangoni convection in acetone: Experiments and comparison with numerical simulations, J. Fluid Mech., 234 (1992), 487-510.

[13] Ezersky, A.B., Garcimartín, A., Mancini, H.L., Pérez-García, Hydrothermal waves in Marangoni convection in a cylindrical container, Phys. Rev. E, 47 (1992), 1126-1131; Spatiotemporal structure of hydrothermal waves in Marangoni convection, Phys. Rev. E, 48 (1992), 4414-4422.

[14] De Saedeleer, C., Garcimartín, A., Chavepeyer, G., Platten, J.K., Lebon, G., The instability of a liquid layer heated from the side when the upper surface is open to air, Phys. Fluids, 8 (1996), 670-676.

[15] Daviaud, F., Vince, J.M., Travelling waves in a fluid layer subjected to a horizontal temperature gradient, Phys. Rev. E, 48 (1993), 4432-4436.

[16] Mukolobwiez, N., Chiffaudel, A., Daviaud, F., Supercritical Eckhaus instability for surface-tension-driven hydrothermal waves, Phys. Rev. Lett., 80 (1998), 4661-4664.

[17] Riley, R.J., Neitzel, G.P., Instability of thermocapillary-buoyancy convection in shallow layers. Part 1. Characterization of steady and oscillatory instabilities, J. Fluid Mech., 359 (1998), 143-164.

[18] Priede, J., Gerbeth, G., Convective, absolute, and global instabilities of thermocapillarybuoyancy convection in extended layers, Phys. Rev. E, 56 (1997), 4187-4199.

[19] Pelacho, M.A., Burguete, J., Temperature oscillations of hydrothermal waves in thermocapillary-buoyancy convection, Phys. Rev. E, 59 (1999), 835-840; Pelacho, M.A., Garcimartín, A., Burguete, J., Local Marangoni number at the onset of hydrothermal waves, Phys. Rev. E, 62 (2000), 477-483; Pelacho, M.A., Ondas hidrotermales en sistemas confinados, Ph.D. Thesis, Universidad de Navarra, 2000.

[20] Burguete, J., Mukolobwiez, N., Daviaud, F., Garnier, N., Chiffaudel, A., Buoyantthermocapillary instabilities in an extended liquid layer subjected to a horizontal temperature gradient, Phys. Fluids, 13 (2001), 2773-2787. 
[21] Garnier, N., Ondes non-linéaires a une et deux dimensions dans une mince couche de fluide, Ph.D. Thesis, Université Paris 7-Denis Diderot, 2000.

[22] Garnier, N., Chiffaudel, A., Two-dimensional hydrothermal waves in an extended cylindrical vessel, Eur. Phys. J. B, 19 (2001), 87-95.

[23] Garnier, N., Chiffaudel, A., Daviaud, F., Prigent, A., Nonlinear dynamics of waves and modulated waves in 1D thermocapillary flows I: General presentation and periodic solutions, Physica D, 174 (2003), 1-29; Garnier, N., Chiffaudel, A., Daviaud, F., Nonlinear dynamics of waves and modulated waves in 1D thermocapillary flows II: Convective/ absolute transition, Physica D, 174 (2003), 30-55.

[24] Shetsova, V.M., Nepomnyashchy, A.A., Legros, J.C., Thermocapillary-buoyancy convection in a shallow cavity heated from the side, Phys. Rev. E, 67 (2003), 066308.

[25] Canuto, C., Hussaini, M.Y., Quarteroni, A., Zang, P.A., Spectral Methods in Fluid Dynamics, Springer, Berlin, 1994.

[26] Briggs, R.J., Electron Streams Interaction with Plasmas, MIT Press, Cambridge, USA, 1964.

[27] Bers, A., Space-time evolution of plasma instabilities-absolute and convective, in Handbook of Plasma Physics, M. Rosenbluth and R.Z. Sagdeev, eds., vol 1, 452-516, NorthHolland, Amsterdam, 1983.

[28] Huerre, P., Monkewitz, P.A., Local and global instabilities in spatially developing flows, Ann. Rev. Fluid Mech., 22 (1990), 473-537.

Paper received: 2004-03-01

Paper accepted: 2004-07-20 